MKG-Chirurg 2017 $\cdot 10: 71$

DOI 10.1007/s12285-017-0099-0

Online publiziert: 3. April 2017

C) Springer Medizin Verlag GmbH 2017

CrossMark

Hans-Peter Ulrich

MKG-Chirurgie LindenArcaden, Lübeck, Deutschland

\title{
10 Jahre Der MKG-Chirurg
}

Sehr verehrte Kolleginnen, sehr geehrte Kollegen,

während unseres Jahreskongresses 2007 in Rostock folgte die Mitgliederversammlung dem Antrag des Vorstands fast einstimmig, die bisherige Zeitschrift der DGMKG - die „MuKi“ - in 2 Zeitschriften zu überführen: eine wissenschaftliche englischsprachige Onlinezeitschrift Oral and Maxillofacial Surgery und eine Mitgliederzeitschrift Der MKG-Chirurg als Organ der DGMKG in Kooperation mit der SGMKG.

Beide Zeitschriften stehen, wie auch zuvor, den österreichischen, schweizerischen und ungarischen Gesellschaften für Mund- Kiefer- und Gesichtschirurgie zur Verfügung.

Die Umsetzung erfolgte für die DGMKG und damit für Sie, unsere Mitglieder, kostenneutral. Ihre damalige Zustimmung wurde von den Herausgebern als Auftrag und Verpflichtung verstanden.

Seit der ersten Ausgabe im Mai 2008 sind jährlich, wie geplant, 4 Ausgaben erschienen. Dies sind mit dieser Ausgabe 448 Beiträge in 37 Ausgaben in 10 Bänden. Darunter findet sich eine große Anzahl an CME-zertifizierten Fortbildungen, Fallvorstellungen in den Rubriken Bild und Fall, Kasuistiken sowie Komplikationen und Gefahren, Journal-ClubBeiträgen, Mitteilungen und Neuem aus dem Medizinrecht.

Die Zahl der Zeitschriftenempfänger ist von 1300 auf über 1500 gestiegen, die Volltextzugriffe im Zeitschriftenarchiv von SpringerLink begannen im Jahre 2008 mit 242 und haben im Jahr 2016 über 16.400 erreicht.

Wir nehmen dies als Nachweis, Ihre Erwartungen erfüllt zu haben.

Dies gelang nur mit der Unterstützung aller Autoren, was umso mehr wiegt, als Der MKG-Chirurg als reines Fortbildungsorgan der DGMKG gegründet wurde. Deshalb an dieser Stelle ganz herzlichen Dank an alle Autoren!

》) Deshalb an dieser Stelle ganz herzlichen Dank an alle Autoren

Danke auch allen Herausgebern und Verantwortlichen, dem wissenschaftlichen Beirat, ohne ihr Engagement und ihre Hilfe wäre Der MKG-Chirurg nicht möglich.

Nicht zuletzt gilt der Dank dem Springer Medizin Verlag, der sich in all den Jahren als zuverlässiger und kompetenter Partner bewährt hat.

Die zurückliegenden erfolgreichen 10 Jahre sind Ansporn für die Zukunft, weiter Ihr Interesse zu wecken und die Unterstützung der Mitglieder und Autoren zu erhalten. Bleiben Sie uns gewogen!

Mit besten kollegialen Grüßen Ihr

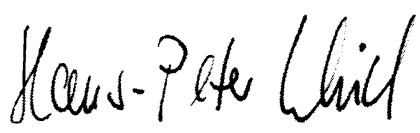

Dr. Dr. Hans-Peter Ulrich

\section{Korrespondenzadresse}

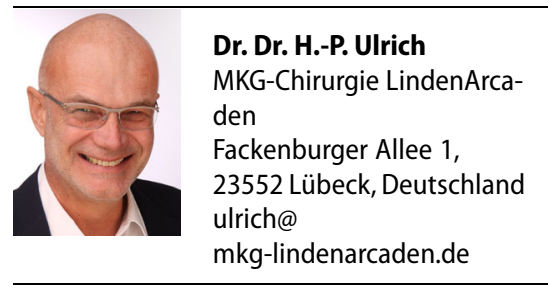

Interessenkonflikt. H.-P. Ulrich gibt an, dass kein Interessenkonflikt besteht. 